\title{
La atención a la diversidad en el aula de idiomas
}

\author{
José Luis Ortega Martín \\ Universidad de Granada
}

\begin{abstract}
RESUMEN: El presente trabajo pretende investigar la atención que se presta a la diversidad del alumnado dentro del aula de idiomas. Aunque las leyes que se aplican actualmente en el sistema educativo (LOGSE y LOCE) contemplan que el profesorado adecue su intervención, la atención al alumno y la evaluación, podemos decir que la realidad es bien distinta pues aunque las intenciones son buenas, el profesorado no se siente capacitado para llevar a cabo esta atención a la diversidad dentro del aula.
\end{abstract}

PALABRAS CLAVE: diversidad, necesidades educativas especiales

\begin{abstract}
This research describes the attention paid by teachers to the diversity of students in the foreign language classroom. The laws currently applied in our educational system (LOGSE and LOCE) establish that teachers must adjust their intervention, their attention to the students, and the evaluation. In practice, that is not what really happens, since, although the teachers are well intentioned, they do not feel confident to address the diversity that they have in their classes.

Key words: diversity, students with special needs
\end{abstract}

\section{INTRODUCCIÓN}

El alumno que presenta necesidades educativas especiales es aquél que encuentra dificultades mayores que las del resto de los alumnos para acceder al currículo propio de su edad, pudiendo estar la razón en una serie de causas internas o debido a un planteamiento educativo inadecuado. Para superar estas carencias, el alumno necesita que se den una serie de condiciones que estén especialmente adaptadas en los diferentes elementos de la propuesta curricular ordinaria así como contar con una serie de recursos específicos distintos de los que la escuela le ofrece diariamente.

La legislación educativa vigente ha rectificado la nomenclatura con la que se refería a este tipo de alumnos, prescindiendo de términos negativos que venían usándose con excesiva frecuencia como los de 'deficientes' o 'minusválidos'. Se entiende que los fines educativos son los mismos para todos los alumnos, con la salvedad de que algunos necesitarán una ayuda especial para alcanzar las metas que se propongan. Así, las necesidades educativas especiales de estos alumnos podrán ser de carácter transitorio o permanente, dependiendo del grado de adecuación que el alumno requiera para el logro de los objetivos propuestos. Además, la situación en nuestro país ha cambiado considerablemente y ahora hemos de estar preparados para acoger a alumnos que presentan necesidades provocadas por su desconocimiento del 
idioma, la cultura, los hábitos, las costumbres religiosas, etc. Los esfuerzos para conseguir la integración de estos alumnos también se han de considerar, como ya se hace, desde la legislación, favoreciendo la formación del profesorado, dotando a los centros de traductores o profesores bilingües que hagan que el alumno no se sienta perdido por razones de idioma, etc.

Hablar de alumnos deficientes es, normalmente, algo negativo y confuso que induce al error, que etiqueta a esos alumnos, y que lleva a pensar que las dificultades de aprendizaje se deben sólo al alumno y sus deficiencias. Emplear ese término resulta poco útil a la hora de planificar y programar las intervenciones educativas de los profesionales que trabajarán con ellos al tiempo que hace creer que la solución se basa en escuelas especiales donde aparcar a estos alumnos. Hablar de alumnos deficientes nos lleva a considerarlos como alumnos incapaces de los que destacamos, sobre todo, sus deficiencias.

$\mathrm{Si}$, por otro lado, hablamos de alumnos con necesidades educativas especiales, estaremos dando un papel más relevante a la preocupación educativa, haciendo evidente que las dificultades de aprendizaje tienen un origen interactivo: alumno-contexto de enseñanza-aprendizaje. Emplear este término nos sitúa en un continuo de necesidades educativas, nos remite al currículo escolar y a los recursos que permiten acceder al mismo. Estas necesidades obligarán a los centros educativos a dotarse de los recursos necesarios con los que dar respuesta a la diversidad, a pensar en el currículo escolar como algo diferente a la concepción tradicional de currículo cerrado en el que el alumno que no alcanza los objetivos ha de repetir o dejar de cursar sus estudios llegada una cierta edad. Llamar a esta situación de necesidades educativas especiales es entender las circunstancias del alumno que, por no llegar o por superar lo establecido en un programa, nos obliga a estudiar lo que podemos hacer para poder ayudarle a conseguir los objetivos y a plantearnos el encontrar una guía para la intervención educativa.

Entendemos que un alumno tiene necesidades educativas especiales cuando presenta dificultades mayores que las de otros para acceder al currículo común de su edad ya sea, como decimos, por causas internas, por dificultades o carencias en su entorno familiar y social o por una historia de aprendizaje desajustada. Este alumno necesita, para compensarlas, una adaptación que se aparte significativamente de la propuesta curricular ordinaria y, si es necesario, también de los medios de acceso al currículo que se ofrecen en los centros escolares.

El profesor de idioma tendrá que prestar especial atención al uso del lenguaje oral y escrito de la lengua extranjera en el aula, pues son instrumentos eficaces de motivación y, por tanto, favorecen, haciendo un uso correcto de los mismos, el que los alumnos más retrasados se incorporen a la marcha de la clase y que los sobredotados no pierdan el tiempo o se aburran. También son instrumentos útiles para aquellos alumnos con necesidades especiales que encontrarán en unos materiales seleccionados cuidadosamente la ayuda necesaria para alcanzar los objetivos que hayan sido propuestos para su caso. El profesor deberá sacar el máximo provecho a la interacción entre los alumnos, las interpretaciones y dramatizaciones de role-plays, lecturas, grabaciones o canciones, la descripción de láminas, fotografías, etc. o, simplemente, a los ejercicios de comprensión oral en los que el alumno tenga que obtener alguna información en particular (Bueno, 2003).

Según Prats (1997: 85), el material curricular más adecuado es el que "facilita el aprendizaje de habilidades intelectuales, el dominio de las técnicas usadas en las disciplinas y el planteamiento de prototipos que simulen la construcción del conocimiento (metodología) de los distintos saberes". En contextos de aula, los medios y recursos didácticos que empleen profesores y alumnos para la enseñanza y aprendizaje son de vital importancia porque condicionan la 
eficacia del programa didáctico y el aprendizaje que experimenta el alumnado. En opinión de Blázquez (1989: 381), los materiales también crean «condiciones para que los maestros y los estudiantes interactúen como seres humanos dentro de un clima donde los hombres dominan el ambiente». En estas situaciones, el material didáctico desempeña un papel crucial ya que:

- Aproxima al alumnado a la realidad de lo que se quiere enseñar.

- Motiva la enseñanza y el aprendizaje, si el material es idóneo y se emplea de forma adecuada.

- Facilita el aprendizaje de conceptos, el desarrollo de procedimientos y estrategias, y la formación de actitudes y valores, relacionados con lo que se enseña y se aprende.

- Representa e ilustra, de forma intuitiva, lo que se explica verbalmente para facilitar su enseñanza y aprendizaje.

- Contribuye a una mejor fijación del aprendizaje.

De ninguna manera tiene sentido hablar de dos grupos de alumnos distintos, los que requieren una atención especial y los que no, necesitando los primeros una educación especial y los segundos no. Además, podríamos plantearnos si se da algún caso entre nuestros alumnos que, a lo largo de su trayectoria educativa, no necesite una atención especial continuada por parte de sus profesores de idioma. Nos parece muy interesante ver cómo se plantea en el Warnock Report (Warnock, 1981) este nuevo planteamiento. En este documento se manifestaba la situación del sistema educativo en el Reino Unido y sirvió como base para actuaciones posteriores:

En lo sucesivo, ningún niño debe ser considerado ineducable: la educación es un bien al que todos tienen derecho. Los fines de la educación son los mismos para todos, independientemente de las ventajas o desventajas de los diferentes niños. Estos fines son, primero, aumentar el conocimiento que el niño tiene del mundo en el que vive, al igual que su comprensión imaginativa, tanto de las posibilidades de ese mundo como de sus propias responsabilidades en él; y, segundo, proporcionarle toda la independencia y autosuficiencia de que sea capaz, enseñándole con este fin lo necesario para que encuentre un trabajo y esté en disposición de controlar y dirigir su propia vida. Evidentemente, los niños encuentran diferentes obstáculos en su camino hacia ese doble fin; para algunos, incluso los obstáculos son tan enormes, que la distancia que recorrerán no será muy larga. Sin embargo, en ellos cualquier progreso es significativo.

(Warnock, 1987: 46-47)

Pero no caigamos en el error de que atender a la diversidad es algo exclusivo de alumnos con síndrome de Down o parálisis de algún tipo. La diversidad también incluye a aquellos alumnos que tienen rasgos de sobredotación, es decir, alumnos para los cuales el currículo ordinario queda corto y necesitan una atención mayor para no caer en el aburrimiento y la desmotivación.

La respuesta a estas necesidades tendrá que venir dada tanto por el profesor de idioma como por el planteamiento curricular que éste haga. También el centro dará una respuesta correcta que debería tener los siguientes rasgos:

1. Un carácter flexible: necesidad, por tanto, de concreción en niveles sucesivos. Las medidas que se adopten nunca se han de considerar como definitivas, sino que habrán de ser adaptadas según la evolución del alumno. 
2. Un carácter abierto de las intenciones educativas, concretado en la generalización en su formulación o, lo que es lo mismo, obviar, en ocasiones, partes del currículo y trabajar otras más adecuadas a sus necesidades. De poco sirve que el profesor se aferre al cumplimiento estricto de todos los puntos contemplados en el programa y sí que manifieste una visión global del mismo, recogiendo de éste lo más significativo y que más pueda ayudar al alumno.

3. Ha de ser amplio en cuanto a los tipos de contenido que contemple.

4. Procurará que se dé una relación estrecha entre las diferentes áreas curriculares, haciendo así que la integración de éstas favorezca la interdisciplinariedad y la adquisición de elementos comunes a varias.

5. La opcionalidad, entendida como la libertad que el profesor tiene para suprimir, añadir, modificar y priorizar contenidos, conceptos, procedimientos, actitudes, etc.

Definimos, pues, la adaptación curricular como la serie de ajustes que se realizan en el currículo con el fin de responder a las necesidades especiales de los alumnos.

El profesor de idioma deberá plantearse ante alumnos de estas características preguntas como qué necesitan aprender, cómo y en qué momento; qué se debe evaluar, cómo y en qué momento, y qué recursos van a ser necesarios para el desarrollo de su proceso de aprendizaje.

El docente tendrá que considerar que las dificultades de aprendizaje del alumno tienen un origen principalmente interactivo, es decir, dependen tanto de las características propias del alumno como de las del entorno en el que se desenvuelve y de la respuesta que se le ofrece. Los ajustes pedagógicos que los responsables de la educación han de llevar a cabo irán en una doble dirección:

a) Adaptaciones de acceso al currículo: modificaciones en cuanto a los aspectos espaciales y materiales de la intervención educativa que permiten que los alumnos con estas necesidades educativas especiales puedan cursar el currículo ordinario.

b) Adaptaciones Curriculares: modificaciones en la programación de los objetivos, contenidos, metodología de actividades y criterios y procedimientos de evaluación para atender la diversidad.

Hablaremos pues de adaptación curricular cuando las modificaciones realizadas lleguen a eliminar algunas de las enseñanzas básicas. Si se llega a dar el caso de que lo que se elimina es un área completa, hablaremos de diversificación curricular (sólo en el segundo ciclo de la ESO).

Otro criterio que permitirá a un profesor, o a cualquier experto, identificar si un alumno tiene necesidades educativas especiales será que precise adaptaciones curriculares significativas, pues las que no lo son se prevén para cualquier alumno en un momento en particular del proceso educativo (refuerzo educativo, que trataremos más adelante). Es precisamente a este tipo de adaptación a la que nos referimos en uno de los items de nuestro cuestionario cuando preguntamos al profesorado su opinión sobre que se adapten los contenidos según su nivel, sin llegar a hablar de adaptaciones curriculares significativas.

Los distintos tipos de adaptación curricular que quedan reflejadas en la Figura 1. 


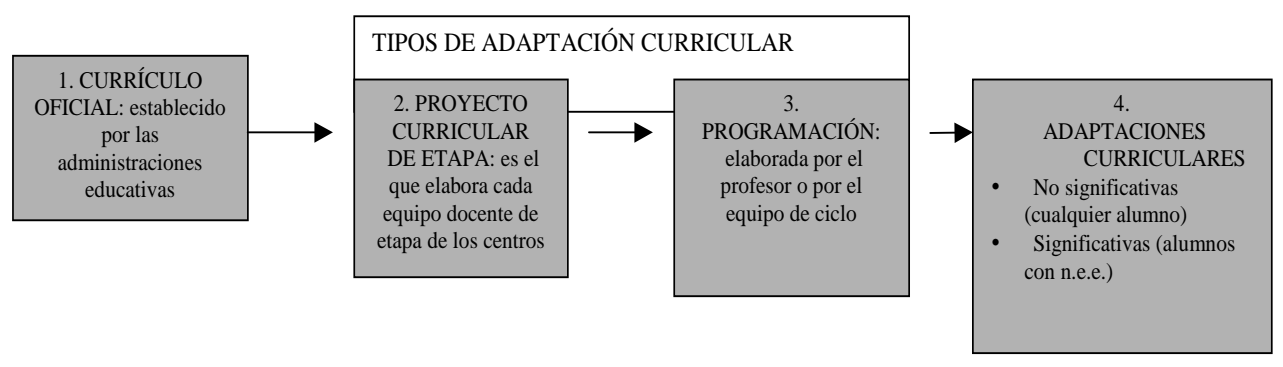

Figura 1. Tipos de adaptación curricular

Dichas adaptaciones podrán tener un mayor o menor grado de concreción, que queda reflejado a continuación:

1. Adaptaciones curriculares de Centro: Se trata del conjunto de modificaciones o ajustes que se realizan en los diferentes elementos de la propuesta educativa que desarrolla elementos de la propuesta educativa que lleva a cabo el equipo docente para el centro - reflejada en el Proyecto Educativo y en los Proyectos Curriculares del Centro - para responder a los alumnos con necesidades educativas especiales, en un continuo de dar respuesta a la diversidad.

En este nivel, las adaptaciones curriculares tratan de:

- Responder a la diversidad de alumnos que tenga el Centro.

- Responsabilizar a todos los profesores en la respuesta educativa a los alumnos con necesidades especiales, participando activamente en la formación de éstos, orientándolos, motivándolos para que se sientan partícipes de la tarea a seguir con estos alumnos, etc.

- Favorecer la integración de los alumnos con necesidades educativas especiales en la dinámica general del centro y las aulas, evitando todo tipo de barreras (físicas o no) que impidan la libre participación de éstos en las actividades y proyectos que se desarrollen.

- Prevenir la aparición o intensificación de las necesidades especiales que puedan producirse como consecuencia de un planteamiento educativo inadecuado, para lo que se establecerán las actuaciones necesarias que evalúen la situación actual, recojan las opiniones de la comunidad educativa, etc., con el fin de no caer en errores de graves consecuencias para la formación del alumnado.

- Favorecer que las adaptaciones individualizadas sean menos significativas, o lo que es lo mismo, que la integración del alumnos sea cada vez mayor y las diferencias menores.

2. En un segundo nivel encontramos las adaptaciones curriculares de aula, es decir, el conjunto de ajustes o modificaciones que se realizan en los diferentes elementos de la propuesta educativa con la que se trabaja con un grupo de alumnos con el fin de facilitar que éstos puedan conseguir sus objetivos -aunque difieran de los del grupocon el mayor grado posible de normalización e integración en la dinámica general del aula. 
El objetivo de las adaptaciones de aula intentan, además de lo señalado para las de Centro, crear las condiciones que posibiliten el desarrollo simultáneo de diferentes programas en el aula (programación del aula, adaptaciones curriculares individualizadas o programas específicos de grupo).

3. En un tercer nivel de concreción se sitúan las adaptaciones curriculares individualizadas, que son el conjunto de ajustes o modificaciones en los diferentes elementos de la propuesta educativa desarrollada para un determinado alumno, que tienen como objetivo -tras una amplia valoración de alumno y contexto- responder a sus necesidades educativas especiales, y que no son comunes al resto de alumnos.

Este tipo de adaptación curricular tiene como objetivos los siguientes:

- Completar y ajustar la respuesta educativa que tanto el centro como el profesor han de elaborar para un alumno con necesidades educativas especiales.

- Responder al principio de individualización de la enseñanza haciendo, como decíamos, que esta adaptación sea individualizada y única para cada alumno que la requiera.

- Las adaptaciones también se pueden concretar en cuanto a las personas que participan activamente en ellas y en los elementos utilizados para llevarlas a cabo (profesor, psicólogo, orientador, pedagogo, materiales adicionales, etc.).

La adaptación no se va a quedar sólo en cómo enseñar sino que va a afectar directamente a la metodología que se ha de emplear y a la selección de actividades que favorezcan la integración de los alumnos. El centro tendrá que velar porque haya unos criterios metodológicos comunes para todos sus alumnos, que se considere la presencia de alumnos con necesidades educativas especiales, programando actividades simultáneas diversas para grupos distintos dentro del aula. También tendrá que conseguir que se usen métodos, técnicas y estrategias específicas adecuadas para el trabajo individual con los alumnos con necesidades educativas especiales.

Se hace por tanto necesario reformular los objetivos de ciclo o etapa para que estos alumnos se incorporen a la marcha de la clase, introduciendo para el aula bloques de contenido especialmente significativos para los alumnos con necesidades educativas especiales y eliminando objetivos generales de ciclo o etapa. La adaptación llegará, incluso, a qué, cuándo y cómo evaluar, estableciendo los criterios de promoción de estos alumnos. Para ello habrá que diseñar actividades de evaluación variadas y utilizar técnicas y procedimientos que resulten de utilidad para todos los alumnos del aula.

Consideramos también que, dentro de la atención a la diversidad, es fundamental que el docente sepa cómo evaluar a sus alumnos, pues de poco servirá un proceso que plantee unos objetivos claros, con procedimientos adecuados y una deficiente evaluación del mismo.

Al respecto, el Decreto de Evaluación de la Junta de Andalucía (Junta de Andalucía, 1995) recoge, en los artículos 15,16 y 17, lo siguiente en cuanto a la evaluación de alumnos con necesidades educativas especiales en los niveles de Primaria y Secundaria.

La primera actuación es el refuerzo educativo como primer paso que el profesor debe dar en su objetivo de favorecer la consecución de los objetivos marcados para el alumno, sin entrar aún en la adaptación. A continuación recurrirá a la adaptación curricular. Se concreta la actuación del docente, que tendrá que eliminar o incluir determinados contenidos para favorecer la consecución de los objetivos planteados en la adaptación. Aparece también la importancia de modificar los criterios de evaluación. Con respecto al tercer punto de este 
mismo artículo, entendemos está bastante alejado de la realidad pues son muchos los alumnos que, aún con adaptaciones curriculares individualizadas significativas, no consiguen alcanzar las capacidades propias de la Educación Primaria. Recordamos que mientras la decisión de adoptar un refuerzo educativo recae sobre el profesor, cuando se lleve a cabo una adaptación curricular significativa, será el Equipo de Orientación Externa (de la correspondiente Delegación de Educación) además del Gabinete de Orientación del propio centro quienes elaboren un informe en el que se recojan las necesidades educativas especiales del alumno y, junto al profesor, se elabore una propuesta curricular específica. Esta propuesta será elevada a la Delegación de Educación que dará o no su aprobación.

Se debe seguir un proceso de observación de la conducta, el trabajo y rendimiento del alumno para plantearnos la aplicación de una adaptación curricular significativa. Las etapas a seguir serán las siguientes:

\begin{tabular}{|c|c|}
\hline & PROCESO DE VALORACIÓN Y ELABORACIÓN DE UNA ADAPTACIÓN CURRICULAR \\
\hline Etapa $1^{\mathrm{a}}$ & $\begin{array}{l}\text { Se observará si el alumno presenta dificultades de aprendizaje en un momento determinado. Si el } \\
\text { problema ya ha sido detectado con antelación, se pasará a la } 4^{\mathrm{a}} \text { etapa. }\end{array}$ \\
\hline Etapa $2^{\mathrm{a}}$ & $\begin{array}{l}\text { Valorar el desarrollo del alumno para determinar los factores que dificultan su aprendizaje. El } \\
\text { profesor realizará los cambios que estén en su mano (refuerzos educativos). }\end{array}$ \\
\hline Etapa $3^{\mathrm{a}}$ & $\begin{array}{l}\text { Observar si las medidas adoptadas han subsanado las insuficiencias detectadas. De ser así, habrá } \\
\text { finalizado el proceso. }\end{array}$ \\
\hline Etapa $4^{\mathrm{a}}$ & $\begin{array}{l}\text { Si las medidas adoptadas no han sido suficientes, habrá que realizar una valoración de: } \\
\text { - El nivel de competencias curriculares } \\
\text { - El estilo de aprendizaje } \\
\text { - El contexto educativo } \\
\text { - La ubicación escolar } \\
\text { - Las orientaciones para la toma de decisiones } \\
\text { - El desarrollo general del alumno } \\
\text { - El contexto familiar y social } \\
\text { Esta valoración será hecha conjuntamente con el equipo de orientación externo e interno del centro. }\end{array}$ \\
\hline Etapa $5^{\mathrm{a}}$ & Determinar, entonces, las necesidades educativas del alumno y decisión de medidas a llevar a cabo. \\
\hline Etapa $6^{\mathrm{a}}$ & Se llevarán a cabo las adaptaciones curriculares significativas o de acceso que sean necesarias. \\
\hline Etapa $7^{\mathrm{a}}$ & Implicación directa no sólo del docente, sino también del centro y la familia. \\
\hline Etapa $8^{\mathrm{a}}$ & Realizar un seguimiento exhaustivo del progreso y desarrollo del alumno. \\
\hline
\end{tabular}


Señalamos, a continuación, los distintos tipos de adaptaciones que se pueden dar en el aula:

Distintos tipos de Adaptaciones Curriculares

\begin{tabular}{|c|c|c|c|}
\hline NIVEL DE ADAPTACIÓN & $\begin{array}{l}\text { DOCUMENTO EN QUE SE } \\
\text { PLASMA }\end{array}$ & $\begin{array}{l}\text { DECISIONES QUE } \\
\text { COMPRENDE }\end{array}$ & AGENTES \\
\hline Adaptación curricular de centro & Proyecto Curricular de Etapa & $\begin{array}{l}\text { * Adaptación del currículo a las } \\
\text { necesidades y características del } \\
\text { medio, centro, alumnado, etc. } \\
\text { * Adaptación en los elementos } \\
\text { de acceso: barreras } \\
\text { arquitectónicas, señalizaciones, } \\
\text { etc. }\end{array}$ & $\begin{array}{l}* \text { Elabora E.T.C.P. } \\
* \text { Aprueba Consejo Escolar } \\
\text { * Supervisado por la Inspección } \\
\text { y Equipos Externos }\end{array}$ \\
\hline Adaptación curricular de aula & Programación de aula & $\begin{array}{l}\text { * Adecuación del P.C.E. A las } \\
\text { características del alumnado del } \\
\text { aula en una secuencia de u.d. }\end{array}$ & $\begin{array}{l}\text { * Elabora equipo de ciclo } \\
* \text { Adapta y desarrolla cada tutor }\end{array}$ \\
\hline $\begin{array}{l}\text { Adaptación dentro de cada } \\
\text { unidad didáctica }\end{array}$ & $\begin{array}{l}\text { Apartados concretos en cada } \\
\text { unidad didáctica }\end{array}$ & $\begin{array}{l}* \text { Refuerzo pedagógico o } \\
\text { educativo } \\
* \text { Adaptaciones metodológicas }\end{array}$ & $\begin{array}{l}* \text { Tutor } \\
* \text { Profesor de apoyo } \\
* \text { Profesor especialista } \\
* \text { Orientador }\end{array}$ \\
\hline $\begin{array}{l}\text { AA.CC. específicas para un } \\
\text { alumno }\end{array}$ & $\begin{array}{l}\text { Adaptaciones curriculares } \\
\text { individualizadas }\end{array}$ & $\begin{array}{l}\text { * Adaptaciones de los elementos } \\
\text { básicos del currículo (objetivos, } \\
\text { áreas, métodos, contenidos, } \\
\text { evaluación) }\end{array}$ & $\begin{array}{l}* \text { Tutor } \\
* \text { Profesores } \\
* \text { Orientador }\end{array}$ \\
\hline Enriquecimiento curricular & $\begin{array}{l}\text { Programa específico dentro de la } \\
\text { programación de aula y/o } \\
\text { apartado complementario dentro } \\
\text { de la a.c.i. }\end{array}$ & $\begin{array}{l}* \text { Desarrollo de programas } \\
\text { complementarios }\end{array}$ & $\begin{array}{l}* \text { Tutor } \\
* \text { Profesores especialistas } \\
* \text { Orientador }\end{array}$ \\
\hline
\end{tabular}

En resumen, podemos señalar las siguientes vías y medidas específicas de atención a la diversidad:

\begin{tabular}{|l|l|}
\hline OPTATIVIDAD & $\begin{array}{l}\text { Contemplada en los objetivos generales de la etapa entendida bien como } \\
\text { mecanismo de refuerzo o ampliación de la oferta educativa }\end{array}$ \\
\hline $\begin{array}{l}\text { ADAPTACIONES } \\
\text { CURRICULARES }\end{array}$ & $\begin{array}{l}\text { Es la acción que se puede dar en el aula para resolver los problemas de } \\
\text { aprendizaje de algunos alumnos (significativas, no significativos, etc.) }\end{array}$ \\
\hline $\begin{array}{l}\text { DIVERSIFICACIÓN } \\
\text { CURRICULAR }\end{array}$ & Se programa para alumnos de ESO y afecta a las áreas completas \\
\hline $\begin{array}{l}\text { PROGRAMAS DE } \\
\text { GARANTÍA SOCIAL }\end{array}$ & $\begin{array}{l}\text { Para los alumnos mayores de 15 años, está estructurado en áreas y } \\
\text { cualifica al alumno para desempeñar una profesión }\end{array}$ \\
\hline
\end{tabular}

\section{OвJetivos}

El objetivo general que nos planteamos en nuestro trabajo es conocer la importancia que dan los docentes a la atención a la diversidad dentro del aula de idioma. Para ello, concretamos los siguientes objetivos:

- conocer si los profesores realmente adaptan los contenidos según los niveles de los alumnos, ya sean éstos mayores o menores que los de la media del grupo,

- si se hacen programaciones específicas para alumnos con necesidades educativas especiales, 
- si se aplica el currículum abierto, centrándose en los intereses de los alumnos,

- si el profesor trabaja según cada situación que se le da en el aula, tras haberla estudiado detenidamente,

- conocer si realmente se trabaja con grupos de diversificación curricular en los niveles superiores de la ESO,

- si se echa de menos profesorado de apoyo para llevar a cabo la atención personalizada que se entiende precisa para estas necesidades especiales,

- conocer qué uso se hace de materiales alternativos al libro de texto,

- si se acude a los gabinetes psicopedagógicos,

- si se lleva a cabo y se usan los resultados de la evaluación inicial como fuente de información para futuras actuaciones.

Los profesores colaboradores en nuestro trabajo han sido agrupados según:
a. Género.
b. Años de docencia.
c. Tipo de centro en el que imparte su docencia.
d. Nivel educativo en el que da clases.
e. Tendencia política.

\section{Metodología}

\subsection{Muestra}

El tipo de muestreo ha sido del tipo no probabilístico casual en el que el criterio de selección de los individuos depende de la posibilidad que hemos tenido de acceder a ellos y que queda distribuido del siguiente modo:

\begin{tabular}{|c|c|c|c|}
\hline \multirow{2}{*}{$\begin{array}{l}\text { PROFESORES } \\
\text { ENCUESTADOS }\end{array}$} & \multicolumn{3}{|c|}{53} \\
\hline \multirow{2}{*}{ Por género } & & total & porcentaje \\
\cline { 2 - 4 } & Mujeres & 30 & $56,6 \%$ \\
\hline \multirow{2}{*}{ Por tipo de centro } & Hombres & 23 & $43,4 \%$ \\
\cline { 2 - 4 } & Público & 20 & $37,7 \%$ \\
\cline { 2 - 4 } & Privado & 7 & $13,2 \%$ \\
\cline { 2 - 4 } & En Prácticas (CAP) & 18 & $34 \%$ \\
\cline { 2 - 4 } & Universidad & 8 & $15,1 \%$ \\
\hline
\end{tabular}




\begin{tabular}{|c|c|c|c|}
\hline \multirow{6}{*}{$\begin{array}{l}\text { Por nivel educativo en el } \\
\text { que imparten docencia }\end{array}$} & $6^{\circ}$ Primaria & 2 & $3,8 \%$ \\
\hline & ESO 1er ciclo & 3 & $5,7 \%$ \\
\hline & $\mathrm{ESO} 2^{\circ}$ ciclo & 10 & $18,9 \%$ \\
\hline & Bachillerato & 12 & $22 \%$ \\
\hline & Universidad & 8 & $15,1 \%$ \\
\hline & En Prácticas (CAP) & 18 & $34,5 \%$ \\
\hline \multirow[t]{2}{*}{ Experiencia docente } & Menos de cinco años & 41 & $77,4 \%$ \\
\hline & Cinco o más años & 12 & $22,6 \%$ \\
\hline \multirow[t]{3}{*}{ Edad } & Menores de 30 años & 38 & $71,7 \%$ \\
\hline & De 31 a 45 años & 12 & $22,6 \%$ \\
\hline & Mayores de 45 años & 3 & $5,7 \%$ \\
\hline \multirow[t]{3}{*}{ Tendencia política } & Izquierda & 15 & $53,6 \%$ \\
\hline & Centro & 6 & $21,4 \%$ \\
\hline & Derecha & 7 & $25 \%$ \\
\hline
\end{tabular}

\subsection{Instrumentos para el control de las variables}

Para nuestro estudio hemos trabajado con un cuestionario elaborado por el autor (véase 6. Anexos) en cuyos once items intentamos recoger la importancia dada a la atención a la diversidad en los procesos de enseñanza y aprendizaje y la valoración que hacen los profesores de la misma. La fiabilidad del cuestionario aplicado se puede apreciar en el siguiente cuadro:

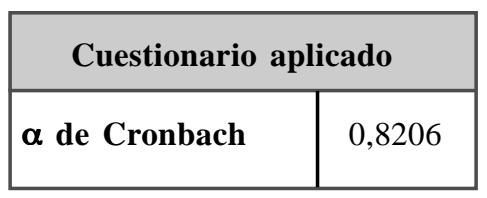

\subsection{Procedimientos}

Hemos intentado recoger en los once items de los que consta el cuestionario utilizado información suficiente para conocer hasta qué punto llega no sólo la implicación de los profesores en cuanto a la actuación con alumnos de necesidades educativas especiales en el aula de idioma, sino también con aquellos alumnos que, sin precisar una adaptación curricular significativa, sí precisan una atención más individualizada. 
En todos los casos se han respetado las normas deontológicas o éticas exigidas en la investigación humana. Así, los sujetos han consentido de modo expreso, consciente y libre a ser investigados. Como investigadores nos hemos ceñido a que el uso de la información obtenida se dirigirá a los fines previos y conocidos por los profesores investigados. Se ha procurado igualmente que de esta investigación tanto alumnos como profesores obtengan el mayor provecho.

El diseño que utilizamos es un procedimiento de investigación cuantitativa no experimental de método descriptivo y correlacional. El método descriptivo de estudios de encuesta que se basa en la formulación de preguntas a personas que poseen la información y la comunican a través de un cuestionario. El método correlacional que pretende describir las relaciones que existen entre las variables que intervienen en un fenómeno.

A continuación indicamos las variables controladas para realizar el estudio en función de los objetivos marcados en el epígrafe 2. Aparecen variables descriptivas (género, tipo de centro en el que imparte, años de docencia y edad, nivel en el que imparte, tendencia política). También aparecen las variables valorativas de cada uno de los aspectos recogidos en el cuestionario. Estas variables se han valorado según una escala Lickert, bajo el siguiente criterio: $1=$ no, nada, nunca, muy mal, nada importante; $2=$ un poco, algo, algunas veces, mal, poco importante; $3=$ lo normal, término medio, aceptable; $4=$ bastante, mucho, casi siempre, bien, importante; 5= muchísimo, siempre, muy bien, muy importante.

Variables descriptivas del profesorado:

- Género:

Variable cualitativa nominal y dicotómica. Se han asignado los siguientes valores: 1 : hombre; 2: mujer.

- Años de docencia:

Variable cualitativa nominal. Se han asignado los siguientes valores: 1: de 1 a 5 años de docencia; 2: más de 5 años de docencia.

- Tipo de centro en el que imparte:

Variable cualitativa nominal. Se han asignado los siguientes valores: 1: titularidad privada; 2: titularidad pública; 3 : universidad.

- Nivel en el que imparte docencia:

Variable cualitativa nominal. Se han asignado los siguientes valores: 1: Primaria; 2: ESO1; 3: ESO2; 4: Bachillerato; 5: Universidad; 6: Profesorado de Prácticas.

- Tendencia política

Variable cualitativa nominal. Se han asignado los siguientes valores: 1: Izquierda; 2: Centro; 3: Derecha.

El procedimiento estadístico ha sido descriptivo. Se ha calculado la fiabilidad (Alfa de Cronbach) y validez factorial para dotar de fiabilidad-validez al cuestionario usado en el estudio, ya que ha sido elaborado por el propio autor. También se han utilizado pruebas como Mann Whitney y Chi-Cuadrado para el estudio estadístico de contraste. Los resultados se expresan en valores medios.

Los datos han sido tratados con el programa SPSS 11.0.1. para Windows. 


\section{Resultados}

a. En cuanto al género de los profesores, los resultados obtenidos han sido los siguientes:

\begin{tabular}{|c|c|c|c|}
\hline Género del Docente & Profesor & Profesora & $\begin{array}{l}\text { Estadístico de } \\
\text { contraste }\end{array}$ \\
\hline v.1 Adaptar los contenidos según el nivel & 4.31 & 4.26 & .882 \\
\hline v.2 Programación específica para los de menor capacidad & 3.80 & 3.50 & .285 \\
\hline v.3 Programación específica para los de mayor capacidad & 3.64 & 3.43 & .402 \\
\hline v.4 Programación específica para alumnos con n.e.e. & 4.08 & 4.33 & .290 \\
\hline v. 5 Currículo abierto & 3.48 & 4.13 & $.003 *$ \\
\hline v.6 No trabaje sobre categorías preestablecidas & 3.70 & 3.74 & .788 \\
\hline v.7 Trabajar con grupos de diversificación curricular & 3.33 & 3.66 & .402 \\
\hline v. 8 Contar con profesorado de apoyo & 4.12 & 4.65 & $.034 *$ \\
\hline v.9 Trabajar con materiales alternativos al libro de texto & 4.15 & 4.65 & $.026 *$ \\
\hline v.10 Acudir al gabinete psicopedagógico & 3.81 & 4.16 & .204 \\
\hline v.11 Realizar evaluación inicial & 4.04 & 4.13 & .753 \\
\hline
\end{tabular}

$* \lesssim 0,05$

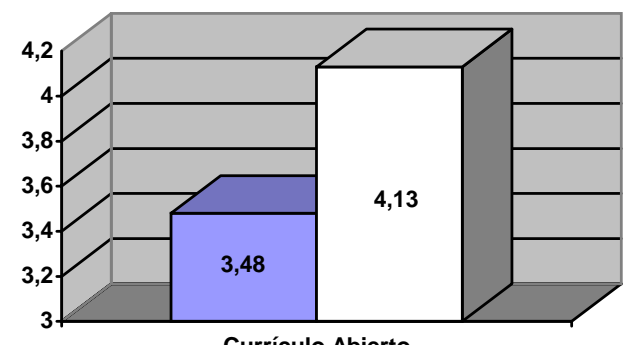

口Hombres $\square$ Mujeres

Currículo Abierto

Observamos que son las profesoras las que creen más conveniente que los profesores el desarrollo del currículo abierto, es decir, se muestran más dispuestas a programar centrándose en los intereses y necesidades del alumnado en lugar de aplicar íntegramente la programación del departamento o seminario.

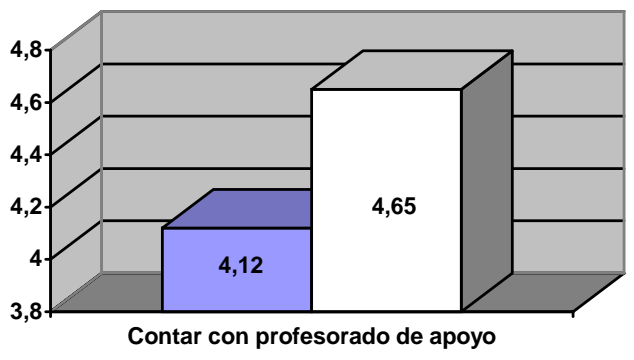

$\square$ Hombres
$\square$ Mujeres


También las profesoras se muestran más a favor de que se cuente con un profesor de apoyo que facilite el trabajo con los alumnos que tengan unas necesidades educativas especiales, pudiendo así prestar una mejor atención a todos los alumnos del grupo.

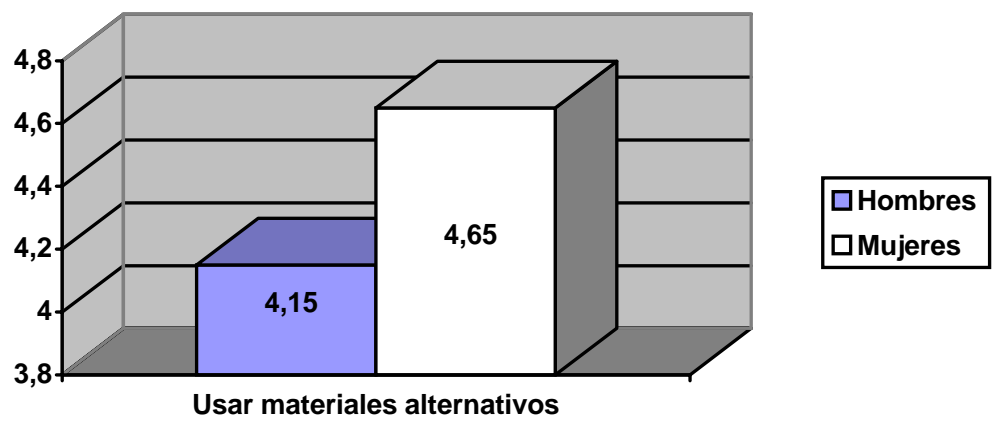

Se aprecian diferencias significativas en v.9 que indican que son las profesoras las que se inclinan más por el uso de materiales alternativos al libro de texto como pueden ser láminas, revistas, periódicos, posters, etc., aplicándolos según el nivel de cada individuo o grupo.

b. Los resultados obtenidos según la experiencia docente de los profesores han sido los siguientes:

\begin{tabular}{|c|c|c|c|}
\hline Experiencia Docente & De 1 a 4 años & De 5 a 30 años & $\begin{array}{c}\text { Estadístico de } \\
\text { contraste }\end{array}$ \\
\hline v.1 Adaptar los contenidos según el nivel & 4.23 & 4.35 & .379 \\
\hline v.2 Programación específica para los de menor capacidad & 3.37 & 3.96 & $.023 *$ \\
\hline v.3 Programación específica para los de mayor capacidad & 3.40 & 3.68 & .231 \\
\hline v.4 Programación específica para alumnos con n.e.e. & 4.10 & 4.36 & .192 \\
\hline v.5 Currículo abierto & 4.00 & 3.64 & .106 \\
\hline v.6 No trabaje sobre categorías preestablecidas & 3.63 & 3.82 & .575 \\
\hline v.7 Trabajar con grupos de diversificación curricular & 3.72 & 3.26 & .198 \\
\hline v.8 Contar con profesorado de apoyo & 4.58 & 4.19 & .342 \\
\hline v.9 Trabajar con materiales alternativos al libro de texto & 4.48 & 4.33 & .605 \\
\hline v.10 Acudir al gabinete psicopedagógico & 4.10 & 3.88 & .433 \\
\hline v.11 Realizar evaluación inicial & 3.97 & 4.22 & .275 \\
\hline
\end{tabular}

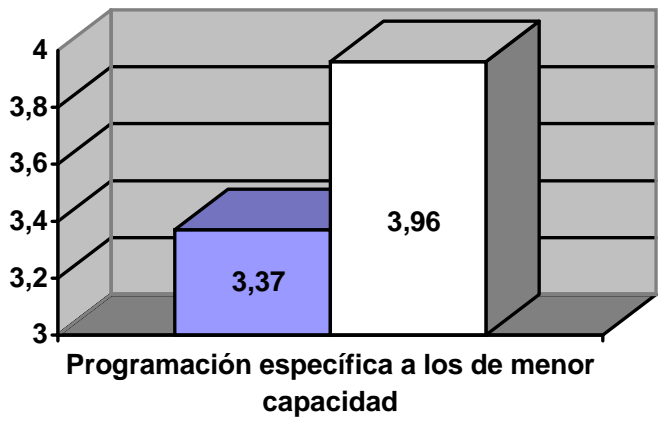


Existen diferencias significativas en v.2 a favor de los profesores noveles, que aparecen como los menos dispuestos a llevar a cabo una programación específica para aquellos alumnos que tienen una menor capacidad, quizás llevados por la creencia de que no es necesario recurrir a medidas extremas. Los más experimentados, sin embargo, sí se muestran claramente a favor de esta medida conocedores, por su puesto, de las ventajas que conlleva tras haber tenido, probablemente, alumnos con estas características.

c. Según el tipo de centro en el que el profesor imparte docencia, los resultados obtenidos han sido los siguientes:

\begin{tabular}{|c|c|c|c|c|c|}
\hline Titularidad del Centro & Titularidad Privada & $\begin{array}{l}\text { Titularidad } \\
\text { Pública }\end{array}$ & Universidad & $\begin{array}{l}\text { Profesorado en } \\
\text { Prácticas }\end{array}$ & $\begin{array}{l}\text { Estadístico de } \\
\text { Contraste }\end{array}$ \\
\hline v.1 Adaptar los contenidos según el nivel. & 4.13 & 4.37 & 4.00 & 4.44 & .584 \\
\hline $\begin{array}{l}\text { v.2 Programación específica para los de menor } \\
\text { capacidad }\end{array}$ & 3.75 & 3.58 & 3.64 & 3.65 & .922 \\
\hline $\begin{array}{l}\text { v.3 Programación específica para los de mayor } \\
\text { capacidad }\end{array}$ & 3.38 & 3.42 & 3.73 & 3.59 & .885 \\
\hline $\begin{array}{l}\text { v.4 Programación específica para alumnos con } \\
\text { n.e.e. }\end{array}$ & 4.13 & 4.37 & 4.18 & 4.12 & .869 \\
\hline v.5 Currículo abierto & 3.25 & 3.90 & 3.69 & 4.11 & .134 \\
\hline v.6 No trabaje sobre categorías preestablecidas & 3.88 & 3.70 & 3.62 & 3.76 & .936 \\
\hline $\begin{array}{l}\text { v.7 Trabajar con grupos de diversificación } \\
\text { curricular }\end{array}$ & 3.50 & 3.50 & 4.17 & 3.00 & .065 \\
\hline v.8 Contar con profesorado de apoyo & 3.63 & 4.60 & 4.55 & 4.44 & .462 \\
\hline $\begin{array}{l}\text { v.9 Trabajar con materiales alternativos al libro } \\
\text { de texto }\end{array}$ & 3.88 & 4.60 & 4.42 & 4.44 & .737 \\
\hline v.10 Acudir al gabinete psicopedagógico & 3,88 & 3.90 & 4.00 & 4.17 & .840 \\
\hline v.11 Realizar evaluación inicial & 3.87 & 4.25 & 3.92 & 4.11 & .526 \\
\hline
\end{tabular}

Aunque no se aprecian diferencias significativas según el tipo de centro en el que los profesores imparten docencia, creemos interesante reflejar la valoración media que éstos hacen, según esta clasificación, de los items recogidos en nuestro cuestionario. Así,

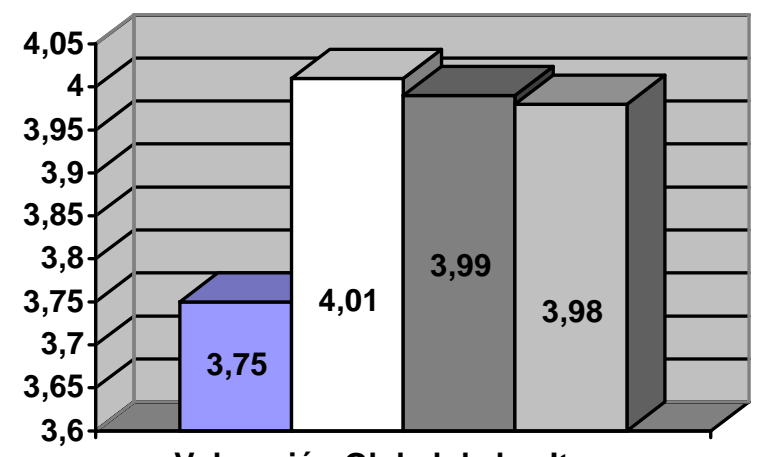

Valoración Global de los Items

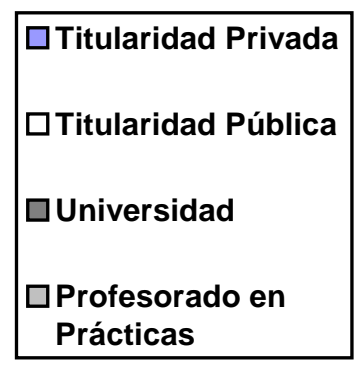

Prácticas 
d. Con respecto al nivel en el que imparte docencia, los resultados han sido los siguientes:

\begin{tabular}{|c|c|c|c|c|c|c|}
\hline Nivel en el que imparte docencia & Primaria & Secundaria & Bachillerato & Universidad & $\begin{array}{c}\text { Profesores en } \\
\text { Prácticas }\end{array}$ & $\begin{array}{c}\text { Estadístico de } \\
\text { Contraste }\end{array}$ \\
\hline $\begin{array}{l}\text { v.1 Adaptar los contenidos según el } \\
\text { nivel }\end{array}$ & 3.33 & 4.31 & 3.44 & 4.00 & 4.33 & .215 \\
\hline $\begin{array}{l}\text { v.2 Programación específica para los de } \\
\text { menor capacidad }\end{array}$ & 2.00 & 3.69 & 4.00 & 4.00 & 3.57 & .199 \\
\hline $\begin{array}{l}\text { v.3 Programación específica para los de } \\
\text { mayor capacidad }\end{array}$ & 1.67 & 3.54 & 3.73 & 4.20 & 3.52 & .141 \\
\hline $\begin{array}{l}\text { v.4 Programación específica para } \\
\text { alumnos con n.e.e. }\end{array}$ & 3.33 & 4.31 & 4.55 & 4.60 & 4.04 & .506 \\
\hline v.5 Currículo abierto & 3.00 & 3.92 & 3.67 & 3.57 & 4.04 & .327 \\
\hline $\begin{array}{l}\text { v.6 No trabaje sobre categorías } \\
\text { preestablecidas }\end{array}$ & 4.00 & 3.92 & 3.50 & 3.57 & 3.74 & .613 \\
\hline $\begin{array}{l}\text { v.7 Trabajar con grupos de } \\
\text { diversificación curricular }\end{array}$ & 2.33 & 3.92 & 3.33 & 3.83 & 3.41 & .187 \\
\hline v.8 Contar con profesorado de apoyo & 2.33 & 4.69 & 4.42 & 4.60 & 4.46 & .302 \\
\hline $\begin{array}{l}\text { v.9 Trabajar con materiales alternativos } \\
\text { al libro de texto }\end{array}$ & 2.67 & 4.62 & 4.58 & 4.33 & 4.46 & .472 \\
\hline $\begin{array}{l}\text { v.10 Acudir al gabinete } \\
\text { psicopedagógico }\end{array}$ & 3.00 & 4.31 & 3.67 & 4.20 & 4.08 & .319 \\
\hline v.11 Realizar evaluación inicial & 4.33 & 4.15 & 4.08 & 3.67 & 4.13 & .759 \\
\hline
\end{tabular}

No se observan diferencias significativas según el nivel en el que imparten docencia los profesores encuestados. Reflejamos, no obstante, la valoración media que hacen de los items recogidos:
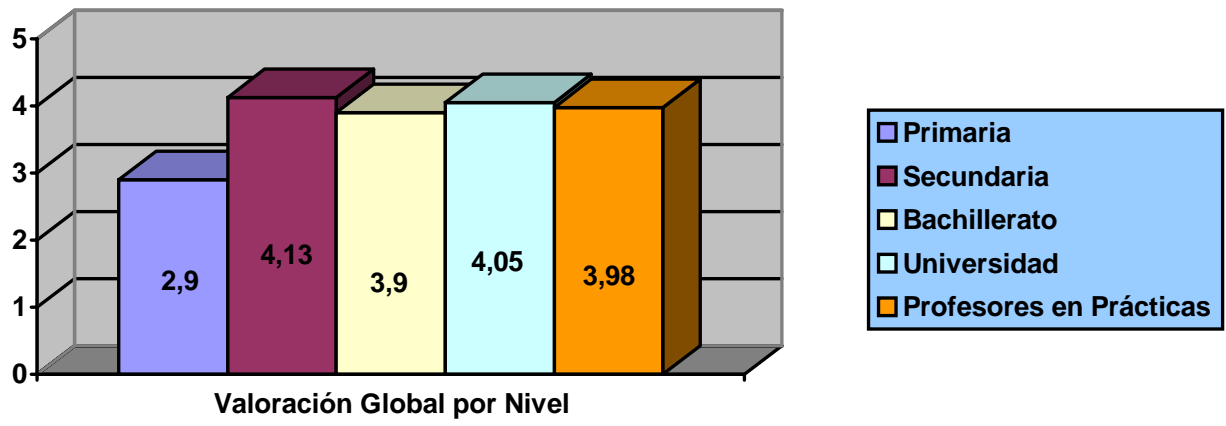
e. También hemos tenido en cuenta la tendencia política manifestada por los docentes, con los siguientes resultados:

\begin{tabular}{|c|c|c|c|c|}
\hline Tendencia Política & Izquierda & Centro & Derecha & $\begin{array}{c}\text { Estadístico de } \\
\text { Contraste }\end{array}$ \\
\hline v.1 Adaptar los contenidos según el nivel & 4.27 & 4.22 & 4.25 & .927 \\
\hline v.2 Programación específica para los de menor capacidad & 4.07 & 3.44 & 4.25 & .300 \\
\hline v.3 Programación específica para los de mayor capacidad & 3.79 & 3.22 & 4.38 & .164 \\
\hline v.4 Programación específica para alumnos con n.e.e. & 4.14 & 4.00 & 4.63 & .267 \\
\hline v.5 Currículo abierto & 3.76 & 3.11 & 3.88 & .109 \\
\hline v.6 No trabaje sobre categorías preestablecidas. & 3.41 & 3.44 & 4.25 & .065 \\
\hline v.7 Trabajar con grupos de diversificación curricular & 3.19 & 3.44 & 3.13 & .856 \\
\hline v.8 Contar con profesorado de apoyo & 4.47 & 3.89 & 4.63 & .799 \\
\hline v.9 Trabajar con materiales alternativos al libro de texto & 4.44 & 3.67 & 4.88 & $.045^{*}$ \\
\hline v.10 Acudir al gabinete psicopedagógico & 4.13 & 3.56 & 4.37 & .178 \\
\hline v.11 Realizar evaluación inicial & 4.06 & 4.22 & 4.13 & .972 \\
\hline
\end{tabular}

$* \leq 0,05$

Hemos observado diferencias significativas en cuanto al trabajo con materiales alternativos al libro de texto según la ideología política manifestada por los propios docentes, con el siguiente resultado:

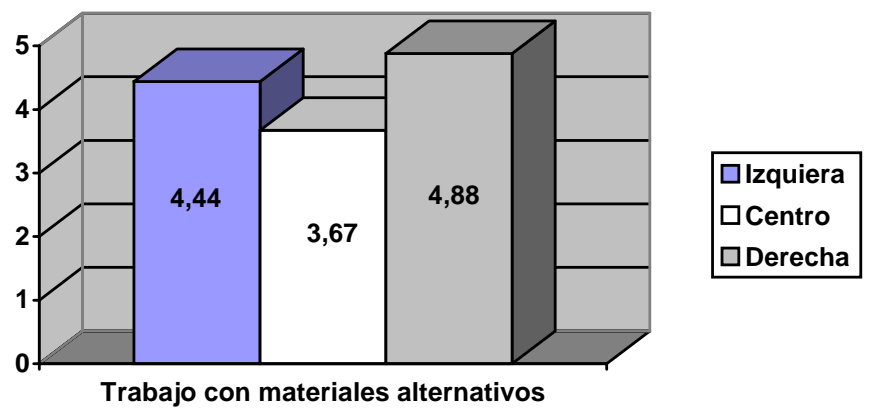

Destacamos también, por último, la valoración global que los profesores hacen, según su tendencia política, de los items recogidos en nuestro cuestionario:

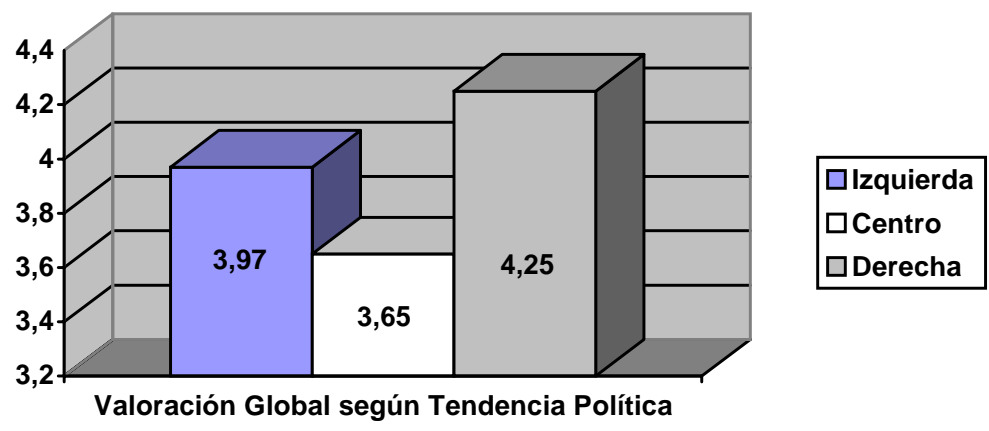




\section{Conclusiones}

En este trabajo nos hemos centrado, principalmente, en la atención a la diversidad dentro del aula de idioma referida a los niveles académicos y el rendimiento del alumno. Nos proponemos, para próximos trabajos, el estudio de los problemas de aprendizaje que se plantean en alumnos inmigrantes causados por el desconocimiento del idioma o las dificultades de adaptación a nuevas costumbres sociales, entre otros factores.

Nuestro principal objetivo era conocer la valoración que hacen los profesores de lo que es la actuación específica dentro del aula de idioma para la mejor atención al alumnado que presenta necesidades educativas especiales. En primer lugar, destacamos que la valoración que los docentes hacen de los items propuestos es muy alta $(0=3,96)$, lo cual indica un interés notable por la atención especializada sobre los alumnos que presentan dificultades de aprendizaje.

Como indicábamos en 2., hemos agrupado las opiniones recogidas según las cinco agrupaciones que definíamos y que nos llevan a concluir lo siguiente:

- En cuanto al género, la valoración por parte de las profesoras $(0=4,05)$ es mayor que la de los profesores $(0=3,86)$, siendo en ambos casos muy alta. Lo que más valoran ambos grupos es el trabajo con materiales alternativos, algo que se entiende, pues, es, para el docente, más fácil preparar materiales con fotocopias, textos adaptados, etc. que perderse en la teoría y la complejidad de la realización de una adaptación curricular. También coinciden en una menor valoración la realización de programas específicos para alumnos de mayor o menor capacidad, lo que de nuevo nos hace pensar que el profesor está dispuesto a trabajar con este tipo de alumnos pero intenta evitar el tener que reflejar por escrito adaptaciones curriculares, programaciones específicas, etc.

- Teniendo en cuenta la experiencia profesional, encontramos también algunos datos muy significativos. Así, los docentes de menor experiencia se muestran más idealistas que los veteranos, decantándose por el currículo abierto y dando más importancia que sus compañeros a la ayuda que puede prestar el gabinete psicopedagógico de cada centro. Los docentes de mayor experiencia, por lo contrario, aceptan tener que elaborar una programación específica para los alumnos con n.e.e. y creen muy conveniente la adaptación de los contenidos según el nivel de los alumnos. Entendemos que esta postura se debe al conocimiento que tienen los profesores más experimentados de la realidad de la clase, que les lleva a reconocer la imposibilidad de seguir un programa estricto y sí a flexibilizarlo cuando sea necesario.

- En lo que respecta al tipo de centro donde los profesores imparten su docencia, creemos significativo que sean los profesores de los centros de titularidad privada los que dan una valoración menor a los factores relacionados con la diversidad y que pretendían recoger una visión generalizada de la actuación con alumnos de n.e.e. en el aula, algo que podría estar vinculado con la denuncia constante que realizan estos docentes sobre la falta de medios de los que disponen (humanos y materiales) para poder llevar a cabo labores específicas con estos alumnos. Por otra parte, creemos conveniente señalar que, mientras los profesores de centros de Primaria, ESO y Bachillerato opinan desde la experiencia de haber tenido alumnos con situaciones especiales en sus aulas, muchos de los profesores de universidad emiten su valoración desde la teoría, pues en estos niveles no se contempla nada referente a actuaciones 
específicas de adaptaciones de programas, salvo casos concretos en los que hay que adaptar un examen a alumnos sordo-mudos, ciegos, etc.

- Si agrupamos las respuestas según el nivel educativo en el que imparten docencia, observamos que la valoración más alta la hacen los profesores de ESO y los universitarios. El orden, de mayor a menor valoración, sería el siguiente: ESO: 0= 4,13; Universidad: $0=4,05$; Profesores en Prácticas: $0=3,98$; Bachillerato: $0=3,90$; Primaria: $0=2,90$. Estos datos nos parecen muy significativos pues vienen a demostrar que el profesor de Primaria es el que ve como algo más normal la presencia de estos alumnos en el aula y el que, al formar parte de su quehacer diario, da menos importancia a adaptaciones, currículos abiertos o profesorado de apoyo; por otro lado, es lógico que sean los profesores de ESO, los que tienen mayor número de alumnos por aula y mayor número de situaciones conflictivas, quienes se muestren más sensibles a cualquier actuación que facilite la normalidad, ya no sólo para el alumno que tiene n.e.e., sino también para el resto del grupo. En Bachillerato, por el contrario, la sensibilización entre el profesorado es menor, pues son menos los alumnos con estas características que cursan este nivel.

- Por último, hemos considerado también la tendencia política de los docentes, con la única intención de buscar alguna posible relación entre aquellos que se consideran más progresistas con actitudes más favorables ante todo lo que sea y suene a atención a la diversidad. Nos sorprende el hecho de que sean los profesores que se reconocen como de centro-derecha / derecha los que hagan una valoración más alta de la diversidad que la que surge de los profesores más progresistas.

Para concluir, queremos dejar claro que nuestro trabajo intenta recoger la opinión de los profesores y no tanto lo que éstos hacen en el aula, para lo que, en investigaciones posteriores, y mediante la observación en ella, podremos comparar lo que los profesores opinan con lo que realmente hacen en el aula. No obstante, creemos que el profesorado de idioma manifiesta una postura ante situaciones de alumnos con n.e.e. en el aula de idioma más positiva que lo que, en definitiva, es su actuación, pues la falta de medios, la masificación de alumnos en algunos casos, la falta de profesorado de apoyo, la enorme burocracia a la que tienen que someterse para elaborar una adaptación curricular, etc., dificultan la realización de estas tareas.

\section{REFERENCIAS}

Blázquez, F. (1989): "Medios didácticos. El medio ambiente y otros recursos para la enseñanza", en Didáctica General, O. Sáenz (dir.). Madrid: Anaya.

Bueno, J. (2003). “Atención a la Diversidad. Inglés. Primaria.” En http://perso.wanadoo.es/jbueno Junta de Andalucía (1995): La Evaluación en la ESO. Materiales Curriculares. Sevilla: Consejería de Educación y Ciencia.

Prats, J. (1977). "El nuevo modelo curricular y la elección del libro de texto", en El libro de texto: materiales didácticos. L. Arranz (coord.). Madrid: Universidad Complutense de Madrid.

Warnock, M. (1987). "Encuentro sobre necesidades de educación especial”. Revista de Educación. Número extraordinario 1987. 


\section{ANEXO \\ Atención a la Diversidad}

Cuestionario del Profesorado

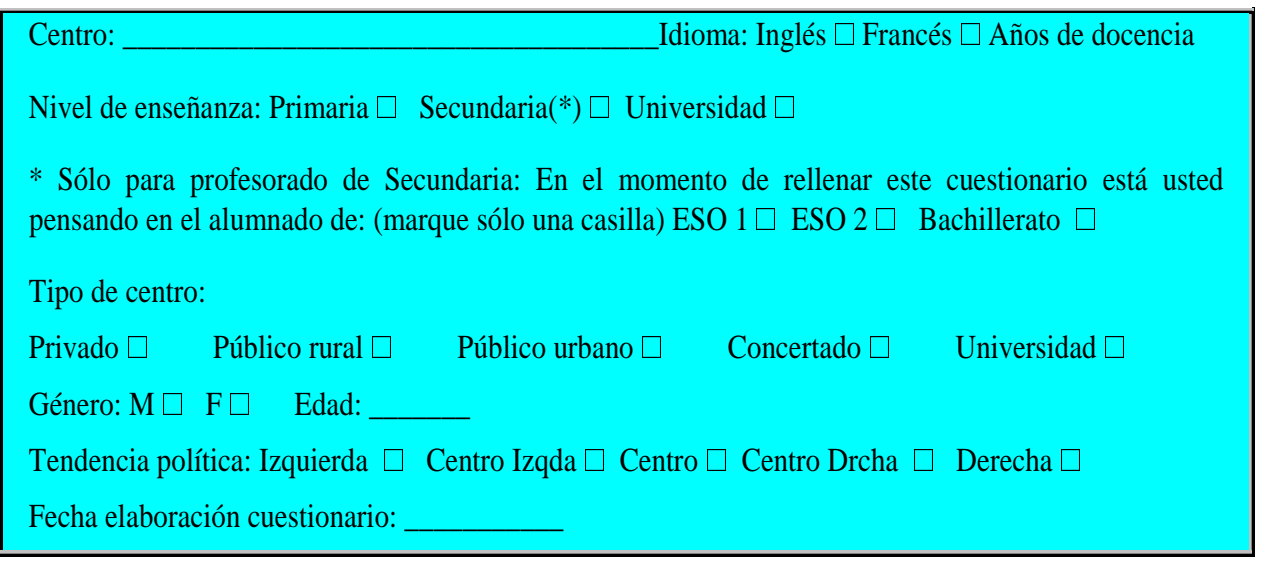

Lea con atención los aspectos siguientes e indique en qué grado los considera adecuados, importantes y útiles para conseguir una enseñanza y aprendizaje de la lengua extranjera eficaces. Indique también la importancia que le asigna a cada punto aunque no lo haya empleado en clase. Puntúe cada aspecto de 1 a 5 aplicando la escala siguiente. Si considera que un ítem no es aplicable en el nivel académico sobre el que está pensando al rellenar cuestionario, escriba N.P. (No Procede) en el espacio punteado:

\begin{tabular}{|l|l|l|l|l|}
\hline 1 & 2 & 3 & 4 & 5 \\
\hline No & Un poco & Lo normal & Bastante & Muchísimo \\
Nada & Algo & Término medio & Mucho & Siempre \\
Nunca & Algunas veces & Normal & Casi siempre & Muy bien \\
Muy mal & Mal & Aceptable & Bien & Muy importante \\
Nada importante & Poco importante & & Importante & \\
\hline
\end{tabular}

\section{ATENCIÓN A LA DIVERSIDAD}

1. (.....) Adaptar los contenidos a los alumnos según su nivel.

2. (.....) Hacer una programación específica para los alumnos que tienen menos capacidad.

3. (.....) Hacer una programación específica para los alumnos que tienen más capacidad.

4. (.....) Hacer una programación específica para los alumnos que tienen necesidades educativas especiales. 
5. (.....) Programar centrándose en los intereses y necesidades del alumnado en lugar de aplicar íntegramente la programación del departamento o seminario (currículum abierto).

6. (.....) No trabajar sobre categorías preestablecidas sino analizar y actuar de un modo distinto y propio sobre cada situación pedagógica.

7. (.....) Trabajar con grupos de diversificación curricular.

8. (.....) Contar con profesorado de apoyo para atender a los alumnos de necesidades educativas especiales.

9. (.....) Trabajar con materiales distintos del libro de texto (láminas, revistas, periódicos, posters, ...) según el nivel de cada individuo o grupo.

10. (.....) Acudir al gabinete psicopedagógico para consultar las razones del fracaso escolar en determinados alumnos.

11. (.....) Realizar la evaluación inicial para conocer la situación de la realidad y el punto de partida. 